This item was submitted to Loughborough's Research Repository by the author.

Items in Figshare are protected by copyright, with all rights reserved, unless otherwise indicated.

\title{
Numerical approximation of a steady MHD problem
}

PLEASE CITE THE PUBLISHED VERSION

http://dx.doi.org/10.1007/978-3-540-75199-1_37

\section{PUBLISHER}

(c) Springer-Verlag Berlin Heidelberg

\section{VERSION}

AM (Accepted Manuscript)

\section{PUBLISHER STATEMENT}

This work is made available according to the conditions of the Creative Commons Attribution-NonCommercialNoDerivatives 4.0 International (CC BY-NC-ND 4.0) licence. Full details of this licence are available at: https://creativecommons.org/licenses/by-nc-nd/4.0/

\section{LICENCE}

CC BY-NC-ND 4.0

\section{REPOSITORY RECORD}

Discacciati, Marco. 2019. "Numerical Approximation of a Steady MHD Problem". figshare. https://hdl.handle.net/2134/18753. 


\title{
Numerical Approximation of a Steady MHD Problem
}

\author{
Marco Discacciati \\ Johann Radon Institute for Computational and Applied Mathematics (RICAM), \\ Altengerberstraße 69, A-4040 Linz, Austria. marco.discacciati@oeaw.ac.at
}

Summary. We consider a magnetohydrodynamic (MHD) problem which models the steady flow of a conductive incompressible fluid confined in a bounded region and subject to the Lorentz force exerted by the interaction of electric currents and magnetic fields. We present an iterative method inspired to operator splitting to solve this nonlinear coupled problem, and a discretization based on conforming finite elements.

\section{Introduction}

MHD studies the interaction of electrically conductive fluids and electromagnetic fields. One of the most interesting aspects of this interaction is the possibility to generate the so-called Lorentz's force, which permits to influence the motion of the fluid in a completely contactless way. With this respect, an important application of MHD occurs in the production of metals.

The mathematical modeling of the processes taking place in such industrial plants is very involved since it requires to take into account many phenomena (multiphase and free-surface flows, electromagnetic fields, temperature effects, chemical reactions, etc.). However, the core model describing the interaction between the liquid metal and the magnetic fields is a nonlinear system formed by Navier-Stokes' and Maxwell's equations coupled by Ohm's law and Lorentz's force. The literature concerning both the mathematical analysis and the finite element approximation of this coupled problem is broad (see, e.g., [5, 7, 8, 9, 10] and references therein).

In this paper, we consider a formulation of a steady MHD problem as a nonlinear coupled system in five unknowns, namely, magnetic field, velocity and pressure of the fluid, electric currents and potential, which presents a "nested" saddle-point structure. Moreover, following the common numerical approach in electromagnetism, we express the magnetic field as the solution of a curl-curl problem, instead of using the Biot-Savart law (see, e.g., [10]).

After briefly discussing the well-posedness of this problem (Sect. 2), we propose and analyze an iterative solution method based on operator-splitting techniques (Sect. 3). In Sect. 4, we present a conforming finite element approximation, and 
we discuss the algebraic form of the iterative schemes. Finally, we present some numerical results (Sect. 5).

\section{Setting and Well-Posedness of the Problem}

We consider a bounded domain $\Omega \subset \mathbb{R}^{3}$ of class $\mathscr{C}^{1,1}$ (see, e.g., [1]), which contains a bounded Lipschitz subdomain $\Omega_{f} \subset \Omega$ filled by an electrically conductive fluid. An external conductor $\Omega_{s}$ is attached to a part of the boundary $\Gamma_{s} \subset \partial \Omega_{f}$, in order to inject an electric current into $\Omega_{f}$. Finally, let $\Omega_{e}$ be an external device which possibly generates a magnetic field $\mathbf{B}_{e}$. A schematic representation of the domain is shown in Fig. 1.

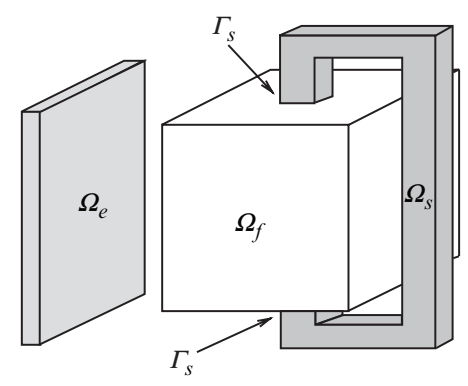

Fig. 1. Schematic representation of the computational setting

In $\Omega_{s}$ we assign an electric current $\mathbf{J}_{s}$ which originates a magnetic field, say $\overline{\mathbf{B}}_{s}$. This current is such that $\operatorname{div} \mathbf{J}_{s}=0$ in $\Omega_{s}, \mathbf{J}_{s} \cdot \mathbf{n}=0$ on $\partial \Omega_{s} \backslash \Gamma_{s}$, and $\mathbf{J}_{s} \cdot \mathbf{n}=j_{s}$ on $\Gamma_{s}$, where $\mathbf{n}$ denotes the unit normal vector directed outward of $\partial \Omega_{f}$. The (known) function $j_{s} \in L^{2}\left(\Gamma_{s}\right)$ fulfills the compatibility condition $\int_{\Gamma_{s}} j_{s}=0$. We suppose that the contact interface $\Gamma_{s}$ between $\Omega_{f}$ and $\Omega_{s}$ is perfectly conductive, i.e.

$$
\mathbf{J}_{s} \cdot \mathbf{n}=j_{s}=\mathbf{J}_{f} \cdot \mathbf{n} \text { on } \Gamma_{s} .
$$

In the fluid domain $\Omega_{f}$ we have a current $\mathbf{J}_{f}$ which generates a magnetic field $\overline{\mathbf{B}}_{f}$. The global magnetic field $\mathbf{B}$ is thus due to the superposition of three components: $\mathbf{B}=\mathbf{B}_{e}+\overline{\mathbf{B}}_{s}+\overline{\mathbf{B}}_{f}$.

The motion of the incompressible conductive fluid in $\Omega_{f}$ is described by the steady Navier-Stokes' equations:

$$
-\eta \triangle \mathbf{u}+\rho(\mathbf{u} \cdot \nabla) \mathbf{u}+\nabla p-\mathbf{J}_{f} \times\left.\mathbf{B}\right|_{\Omega_{f}}=\mathbf{0}, \quad \operatorname{div} \mathbf{u}=0 \quad \text { in } \Omega_{f},
$$

where $\mathbf{u}$ and $p$ are the velocity and the pressure of the fluid, respectively, while $\eta, \rho>0$ are the fluid viscosity and density. We supplement (2) with the Dirichlet boundary condition $\mathbf{u}=\mathbf{g}$ on $\partial \Omega_{f}$, $\mathbf{g}$ being an assigned velocity field such that $\int_{\partial \Omega_{f}} \mathbf{g} \cdot \mathbf{n}=0 . \mathbf{J}_{f} \times\left.\mathbf{B}\right|_{\Omega_{f}}$ is the Lorentz force exerted on the fluid by the interaction of the magnetic field $\mathbf{B}$ and the electric current $\mathbf{J}_{f}$. 
Finally, the electric current $\mathbf{J}_{f}$ satisfies

$$
\sigma^{-1} \mathbf{J}_{f}+\nabla \phi-\mathbf{u} \times\left.\mathbf{B}\right|_{\Omega_{f}}=\mathbf{0}, \quad \operatorname{div} \mathbf{J}_{f}=0 \quad \text { in } \Omega_{f},
$$

where $\phi$ is the electric potential and $\sigma>0$ is the electric conductivity of the fluid. We impose the boundary condition (1) on $\Gamma_{s}$, while we set $\mathbf{J}_{f} \cdot \mathbf{n}=0$ on $\partial \Omega_{f} \backslash \Gamma_{s}$.

In order to give a more useful representation of the magnetic field $\mathbf{B}$, we consider the divergence-free extension $\mathbf{E}_{s} j_{s}$ of $j_{s} . \mathbf{E}_{s}$ is a continuous extension operator $\mathbf{E}_{s}$ : $L^{2}\left(\Gamma_{s}\right) \rightarrow \boldsymbol{H}\left(\operatorname{div} ; \Omega_{f}\right)$, such that $\mathbf{E}_{s} j_{s} \cdot \mathbf{n}=0$ on $\partial \Omega_{f} \backslash \Gamma_{s}, \mathbf{E}_{s} j_{s} \cdot \mathbf{n}=j_{s}$ on $\Gamma_{s}$, and $\operatorname{div}\left(\mathbf{E}_{s} j_{s}\right)=0$ in $\Omega_{f}$ (see [2]). Then, we decompose $\mathbf{J}_{f}=\mathbf{J}_{0}+\mathbf{E}_{s} j_{s}$, with $\mathbf{J}_{0} \in \boldsymbol{H}\left(\operatorname{div} ; \Omega_{f}\right), \mathbf{J}_{0} \cdot \mathbf{n}=0$ on $\partial \Omega_{f}$.

Now, let us consider the currents

$$
\overline{\mathbf{J}}_{s}=\left\{\begin{array}{ll}
\mathbf{E}_{s} j_{s} & \text { in } \Omega_{f}, \\
\mathbf{J}_{s} & \text { in } \Omega_{s}, \\
\mathbf{0} & \text { in } \Omega \backslash\left(\bar{\Omega}_{f} \cup \bar{\Omega}_{s}\right),
\end{array} \quad \text { and } \quad \overline{\mathbf{J}}_{0}= \begin{cases}\mathbf{J}_{0} & \text { in } \Omega_{f}, \\
\mathbf{0} & \text { in } \Omega \backslash \bar{\Omega}_{f},\end{cases}\right.
$$

where $\overline{\mathbf{J}}_{0}$ satisfies $\operatorname{div} \overline{\mathbf{J}}_{0}=0$ in $\Omega$ and $\overline{\mathbf{J}}_{0} \cdot \mathbf{n}=0$ on $\partial \Omega$. Then, the magnetic fields $\mathbf{B}_{s}$ and $\mathbf{B}_{0}$ generated by $\overline{\mathbf{J}}_{s}$ and $\overline{\mathbf{J}}_{0}$, respectively, can be represented as the solution of the problems:

$$
\begin{aligned}
& \operatorname{curl}\left(\mu^{-1} \mathbf{B}_{s}\right)=\overline{\mathbf{J}}_{s} \text { in } \Omega, \\
& \operatorname{div} \mathbf{B}_{s}=0 \quad \text { in } \Omega \\
& \mathbf{B}_{s} \cdot \mathbf{n}=0 \quad \text { on } \partial \Omega \text {, } \\
& \text { and } \\
& \operatorname{curl}\left(\mu^{-1} \mathbf{B}_{0}\right)=\overline{\mathbf{J}}_{0} \text { in } \Omega, \\
& \operatorname{div} \mathbf{B}_{0}=0 \quad \text { in } \Omega \text {, } \\
& \mathbf{B}_{0} \cdot \mathbf{n}=0 \quad \text { on } \partial \Omega \text {. }
\end{aligned}
$$

$\mu>0$ is the magnetic permeability that we assume to be constant in $\Omega$.

The usual approach to compute $\mathbf{B}_{0}$ is to introduce a vector potential $\mathbf{A}$ such that curl $\mathbf{A}=\mathbf{B}_{0}$, and to reformulate the corresponding problem (5) as

$$
\begin{aligned}
\operatorname{curl}\left(\mu^{-1} \operatorname{curl} \mathbf{A}\right)+\varepsilon \mathbf{A} & =\overline{\mathbf{J}}_{0} & & \text { in } \Omega, \\
\mathbf{A} \times \mathbf{n} & =\mathbf{0} & & \text { on } \partial \Omega .
\end{aligned}
$$

Remark that the boundary condition $\mathbf{A} \times \mathbf{n}=\mathbf{0}$ on $\partial \Omega$ implies $\mathbf{B}_{0} \cdot \mathbf{n}=0$ on $\partial \Omega$, and that the perturbation term of order $O(\varepsilon)$, with $0<\varepsilon \ll 1$, has been added to guarantee the uniqueness of the solution $\mathbf{A}$, which otherwise would be defined only up to gradients of arbitrary scalar functions.

Using these notations, we can rewrite the magnetic field $\mathbf{B}$ as $\mathbf{B}=\mathbf{B}_{e}+\mathbf{B}_{s}+$ curl $\mathbf{A}$, A being the only unknown component which depends on the unknown current $\mathbf{J}_{0}$.

Notice that the MHD problem (2), (3) and (6) would be nonlinear even if we considered instead of (2) the Stokes equations:

$$
-\eta \triangle \mathbf{u}+\nabla p-\mathbf{J}_{f} \times\left.\mathbf{B}\right|_{\Omega_{f}}=\mathbf{0}, \quad \operatorname{div} \mathbf{u}=0 \quad \text { in } \Omega_{f} .
$$

Indeed, there is an intrinsic nonlinearity due to the coupling terms $\mathbf{J}_{f} \times\left.\mathbf{B}\right|_{\Omega_{f}}$ and $\mathbf{u} \times\left.\mathbf{B}\right|_{\Omega_{f}}$. For simplicity, we illustrate our solution method avoiding for the moment the nonlinearity due to the convection term $(\mathbf{u} \cdot \nabla) \mathbf{u}$ in $(2)$. Thus, from now on, we regard (7), (3), (6) as our MHD problem.

The well-posedness of the MHD system can be proved using the Banach Contraction Theorem. In particular, we can state the following result (see [2]). 
Proposition 1. Assume that $\mathbf{B}_{e \mid \Omega_{f}} \in\left(L^{3}\left(\Omega_{f}\right)\right)^{3}$. If the physical parameters $\mu^{-1}$, $\eta, \sigma^{-1}$ are sufficiently large, whereas the boundary data $\mathbf{g} \in\left(H^{1 / 2}\left(\partial \Omega_{f}\right)\right)^{3}, j_{s} \in$ $L^{2}\left(\Gamma_{s}\right)$ and the assigned magnetic field $\mathbf{B}_{e}$ are small enough, the MHD problem has a unique solution $\mathbf{A} \in \boldsymbol{H}(\mathbf{c u r l} ; \Omega), \mathbf{u} \in\left(H^{1}\left(\Omega_{f}\right)\right)^{3}, p \in L_{0}^{2}\left(\Omega_{f}\right), \mathbf{J}_{f} \in \boldsymbol{H}\left(\operatorname{div} ; \Omega_{f}\right)$, $\phi \in L_{0}^{2}\left(\Omega_{f}\right)$.

The conditions imposed on the physical parameters are required to ensure the existence of a solution and its uniqueness. From the proof in [2], we can see that the larger the viscosity $\eta$ of the fluid and the smaller its conductivity $\sigma$, the larger the boundary data $\mathbf{g}$ and $j_{s}$ may become.

\section{Iterative Solution Methods}

In this section, we consider possible methods to compute the solution of the MHD problem by independently solving its fluid and magnetic-field subproblems. In particular, we first compute curl $\mathbf{A}$ in $\Omega$, and then we linearize (7) and (3) in $\Omega_{f}$ using the magnetic field just obtained. The latter problems can be solved separately or in a coupled fashion. Ad-hoc solution techniques known in literature may be used to deal with each subproblem, and "reusage" of existing specific codes may be envisaged as well.

Precisely, we propose the following algorithm.

Consider an initial guess $\mathbf{J}_{0}^{(0)}$ for the electric current in $\Omega_{f}$, and set $\overline{\mathbf{J}}_{0}^{(0)}$ as in (4). For $k \geq 0$, until convergence,

1. solve (6) with $\overline{\mathbf{J}}_{0}^{(k)}$ as right-hand side to compute $\mathbf{A}^{(k)}$.

2. Then, solve the problem in $\Omega_{f}$ using one of the following strategies:

2a. Coupled approach: solve in $\Omega_{f}$ the system

$$
\begin{aligned}
-\eta \triangle \mathbf{u}^{(k+1)}+\nabla p^{(k+1)}-\left(\mathbf{J}_{0}^{(k+1)}+\mathbf{E}_{s} j_{s}\right) \times\left(\mathbf{B}_{e}+\mathbf{B}_{s}+\operatorname{curl} \mathbf{A}^{(k)}\right) & =\mathbf{0}, \\
\operatorname{div} \mathbf{u}^{(k+1)} & =0, \\
\sigma^{-1}\left(\mathbf{J}_{0}^{(k+1)}+\mathbf{E}_{s} j_{s}\right)+\nabla \phi^{(k+1)}-\mathbf{u}^{(k+1)} \times\left(\mathbf{B}_{e}+\mathbf{B}_{s}+\mathbf{c u r l} \mathbf{A}^{(k)}\right) & =\mathbf{0}, \\
\operatorname{div}\left(\mathbf{J}_{0}^{(k+1)}+\mathbf{E}_{s} j_{s}\right) & =0 .
\end{aligned}
$$

2b. Split approach: solve first the Stokes problem (8)-(9) in $\Omega_{f}$, taking $\mathbf{J}_{0}^{(k)}$ instead of $\mathbf{J}_{0}^{(k+1)}$. Then, using the velocity field $\mathbf{u}^{(k+1)}$ just computed, solve (10)-(11).

3. In both cases $2 \mathrm{a} / \mathrm{b}$, define the electric current at the successive step possibly considering a relaxation: $\mathbf{J}_{0}^{(k+1)} \leftarrow \theta \mathbf{J}_{0}^{(k+1)}+(1-\theta) \mathbf{J}_{0}^{(k)}, 0<\theta \leq 1$.

Under the same hypotheses of Proposition 1, we can show that there exists a positive radius $\rho_{J}>0$ such that $\mathbf{J}_{0}^{(k)}$ converges with respect to the $L^{2}$-norm in the ball $B_{J}=\left\{\mathbf{J} \in \boldsymbol{H}\left(\operatorname{div} ; \Omega_{f}\right):\|\mathbf{J}\|_{L^{2}\left(\Omega_{f}\right)} \leq \rho_{J}\right\}$ (see $\left.[2]\right)$. 


\section{Conforming Finite Element Approximation}

Let $\mathcal{T}_{h}$ be a regular triangulation of $\bar{\Omega}$ made up of tetrahedra, such that the triangulations induced on $\Omega \backslash \bar{\Omega}_{f}$ and $\Omega_{f}$ are compatible on $\partial \Omega_{f}$.

We discretize the MHD system considering the $\boldsymbol{H}$ (curl; $\Omega$ )-conforming Nédélec elements to approximate the vector potential $\mathbf{A}$, the $\boldsymbol{H}$ (div; $\Omega_{f}$ )-conforming RaviartThomas elements for the electric current and potential, and the Taylor-Hood elements for the Stokes problem (see, e.g., [11] for a presentation of these spaces). Thanks to the inf-sup stability enjoyed by the Raviart-Thomas and the Taylor-Hood elements, it can be proved that also this compound finite element approximation is inf-sup stable, without any further compatibility requirement (see [2]).

Finally, remark that using Raviart-Thomas and Nédélec elements we can deal in a more natural way also with non-convex polyhedral domains, where the magnetic field is in general not in $H^{1}$, and it would be erroneously represented by elements of Lagrangian type.

Let us now briefly consider the algebraic form of step 2 in the algorithm of Sect. 3.

After computing the discrete field $\mathbf{A}_{h}^{(k)}$ ( $h$ denotes finite element approximations), we assemble the following matrix which realizes at the discrete level the coupling between the fluid and the electric-current problems in $\Omega_{f}$ :

$$
\mathrm{C}_{i j}^{(k)}=-\int_{\Omega_{f}}\left[\mathbf{J}_{h}^{i} \times\left(\mathbf{B}_{e}^{h}+\mathbf{B}_{s}^{h}+\operatorname{curl} \mathbf{A}_{h}^{(k)}\right)\right] \cdot \mathbf{v}_{h}^{j} .
$$

$\left(\mathbf{J}_{h}^{i}\right.$ and $\mathbf{v}_{h}^{j}$ denote basis functions for the discrete spaces of the electric currents and fluid velocity, respectively.)

Then, in the coupled approach $2 \mathrm{a}$, one has to solve the $4 \times 4$ block linear system:

$$
\left(\begin{array}{cccc}
\mathrm{A} & \mathrm{B}^{T} & \left(\mathrm{C}^{(k)}\right)^{T} & 0 \\
\mathrm{~B} & 0 & 0 & 0 \\
-\mathrm{C}^{(k)} & 0 & \mathrm{D} & \mathrm{E}^{T} \\
0 & 0 & \mathrm{E} & 0
\end{array}\right)\left(\begin{array}{c}
\mathbf{u}_{h}^{(k+1)} \\
p_{h}^{(k+1)} \\
\mathbf{J}_{0, h}^{(k+1)} \\
\phi_{h}^{(k+1)}
\end{array}\right)=\mathbf{f},
$$

whose matrix presents a "nested" saddle-point structure.

On the other hand, the decoupled strategy $2 \mathrm{~b}$ requires to solve first the linear system (which corresponds to the Stokes problem):

$$
\left(\begin{array}{ll}
\mathrm{A} & \mathrm{B}^{T} \\
\mathrm{~B} & 0
\end{array}\right)\left(\begin{array}{l}
\mathbf{u}_{h}^{(k+1)} \\
p_{h}^{(k+1)}
\end{array}\right)=\left(\begin{array}{c}
-\left(\mathrm{C}^{(k)}\right)^{T} \mathbf{J}_{0, h}^{(k)} \\
\mathbf{0}
\end{array}\right)+\text { boundary terms, }
$$

and then the system associated to (3):

$$
\left(\begin{array}{ll}
\mathrm{D} & \mathrm{E}^{T} \\
\mathrm{E} & 0
\end{array}\right)\left(\begin{array}{l}
\mathbf{J}_{0, h}^{(k+1)} \\
\phi_{h}^{(k+1)}
\end{array}\right)=\left(\begin{array}{c}
\mathrm{C}^{(k)} \mathbf{u}_{h}^{(k+1)} \\
\mathbf{0}
\end{array}\right)+\text { boundary terms. }
$$

Thus, an iteration (13)-(14) corresponds to a Gauss-Seidel step for (12). 


\section{Numerical Results}

We consider a rectangular domain $\Omega_{f}$ between two parallel conductive wires at 0.25 $\mathrm{m}$ from its lateral walls and bottom surface (Fig. 2, left). An electric current in the wires originates a magnetic field $\mathbf{B}_{e}$ (Fig. 2, right). We impose $\mathbf{u}=\mathbf{0}$ on $\partial \Omega_{f}$, $\mathbf{J}_{f} \cdot \mathbf{n}=0$ on the lateral boundary, while we assign $\mathbf{J}_{f} \cdot \mathbf{n}=20$ A on the top and bottom surfaces. The physical parameters are chosen to represent a melted metal.
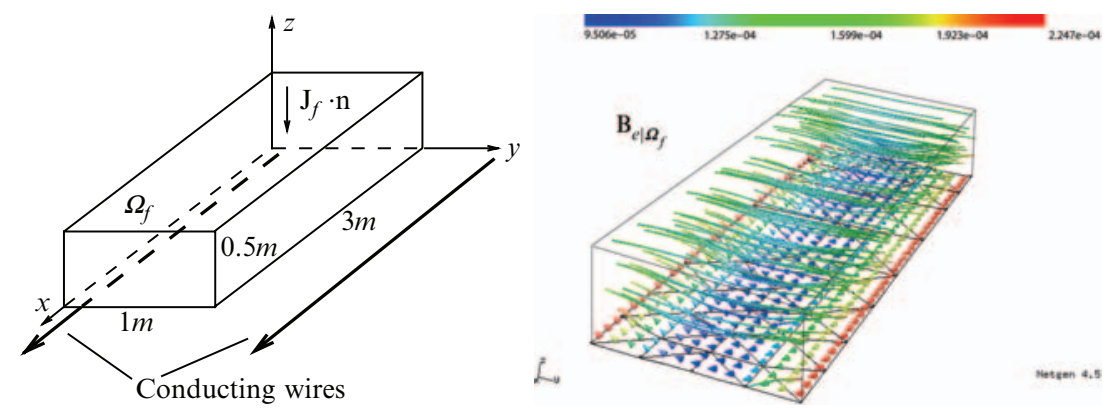

Fig. 2. Schematic representation of the setting (left), and restriction of the field lines of the magnetic field $\mathbf{B}_{e}$ to $\Omega_{f}$ (right)

We consider two uniform computational meshes made of tetrahedra, and few possible choices of the degree of the polynomials used for the finite element approximation. We apply the decoupled algorithm $2 \mathrm{~b}$, using a relaxation with $\theta=0.4$. The stopping criterion is based on the relative increment of the unknown $\mathbf{J}_{f}$, with tolerance $10^{-5}$. The implementation has been done within NGSolve (http://www.hpfem.jku.at/ngsolve/), while the linear systems have been solved using the direct solver PARDISO

(http://www.computational.unibas.ch/cs/scicomp/software/pardiso/).

As shown in Fig. 3, the interaction between the magnetic fields and the electric current $\mathbf{J}_{f}$ gives rise to a double symmetric rotational movement of the fluid with larger velocity towards the bottom of $\Omega_{f}$. The iterations required to convergence are reported in table 1 . We observe that they remain bounded and essentially independent of the number of unknowns.

\section{Conclusions and Perspectives}

The preliminary numerical results obtained using the decoupled iterative scheme seem quite promising. However, a more thorough analysis of the convergence rate is in order, especially concerning the dependence on the mesh size $h$ and on the degree of the polynomials used. Moreover, we are investigating effective techniques for the saddle-point problem (12) (see [4]), with particular interest in applications to optimal control, where problems with a similar structure are quite often encountered when imposing the optimality (Karush-Kuhn-Tucker) conditions (see [3, 6]). 
Acknowledgement. The support of the Austrian Academy of Science is gratefully acknowledged.

\section{References}

[1] R.A. Adams. Sobolev Spaces. Academic Press, New York, 1975.

Table 1. Convergence results for two computational grids and different choices of the degrees of the finite elements

\begin{tabular}{ccccc}
\hline order of Nédélec FE & 1 & 2 & 1 \\
order of Taylor-Hood FE & 2 & 2 & 2 \\
order of Raviart-Thomas FE & 1 & 1 & 2 \\
\hline Grid 1 & dofs & 6071 & 9018 & 11543 \\
(344 tetrahedra) & iterates & 22 & 22 & 22 \\
Grid 2 & dofs & 42602 & 64224 & 85130 \\
(2752 tetrahedra) & iterates & 22 & 22 & 22 \\
\hline
\end{tabular}

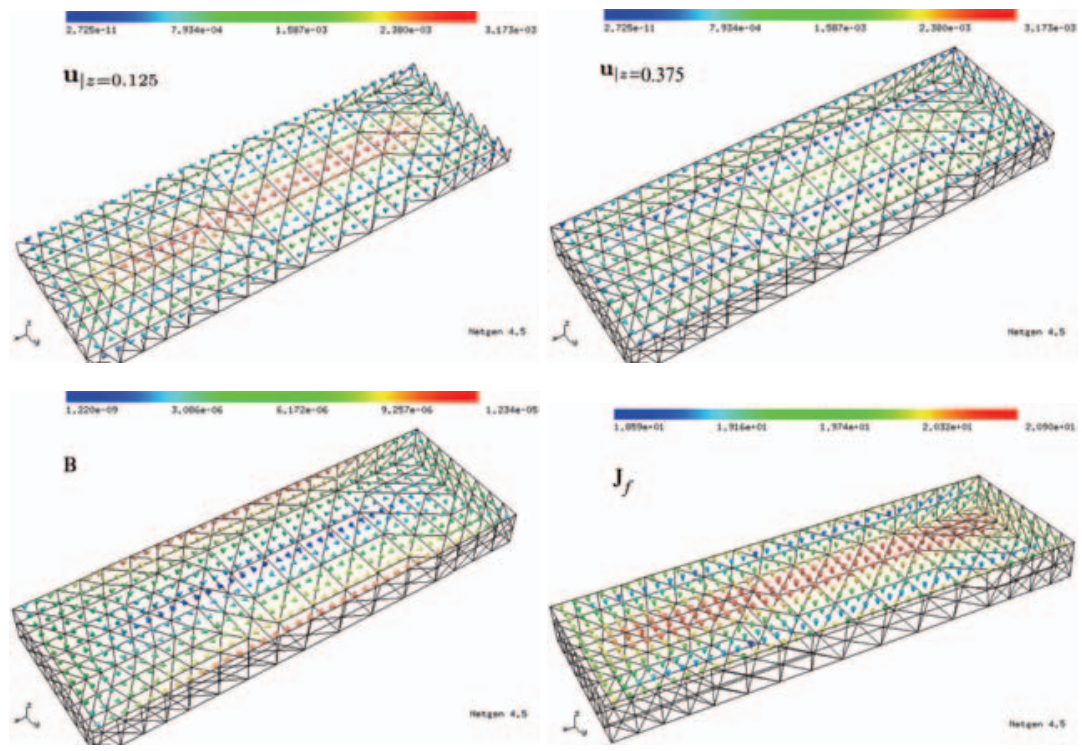

Fig. 3. Computed velocity field across the plane $z=0.125$ (top left) and $z=0.375$ (top right), total magnetic field $\mathbf{B}$ (bottom left), and electric current $\mathbf{J}_{f}$ (bottom right) 
[2] M. Discacciati. Mathematical and numerical analysis of a stready magnetohydrodynamic problem. Technical Report 38, RICAM, Linz, 2006.

[3] M. Discacciati and R. Griesse. Finite element solution of a stationary optimal control problem in magnetohydrodynamics: Stokes case. Technical report, RICAM, Linz, 2007. In preparation.

[4] M. Discacciati and W. Zulehner. Preconditioning techniques for a saddle-point problem arising in magnetohydrodynamics. Technical report, RICAM, Linz, 2007. In preparation.

[5] J.F. Gérbeau, C. Le Bris, and T. Lelièvre. Mathematical Methods for the Magnetohydrodynamics of Liquid Metals. Oxford Science Publications, New York, 2006.

[6] R. Griesse and K. Kunisch. Optimal control for a stationary MHD system in velocity-current formulation. SIAM J. Control Optim., 45(5):1822-1845, 2006.

[7] J.L. Guermond and P.D. Minev. Mixed finite element approximation of an MHD problem involving conducting and insulating regions: the 3D case. Numer. Methods Partial Differential Equations, 19(6):709-731, 2003.

[8] M.D. Gunzburger, A.J. Meir, and J.S. Peterson. On the existence, uniqueness, and finite element approximation of solutions of the equations of stationary, incompressible magnetohydrodynamics. Math. Comp., 56(194):523-563, 1991.

[9] W.J. Layton, A.J. Meir, and P.G. Schmidt. A two-level discretization method for the stationary MHD equations. Electr. Trans. Numer. Anal., 6:198-210, 1997.

[10] A.J. Meir and P.G. Schmidt. Analysis and numerical approximation of a stationary MHD flow problem with nonideal boundary. SIAM J. Numer. Anal., 36(4):1304-1332, 1999.

[11] P. Monk. Finite Element Methods for Maxwell's Equations. Oxford Science Publications, New York, 2003. 